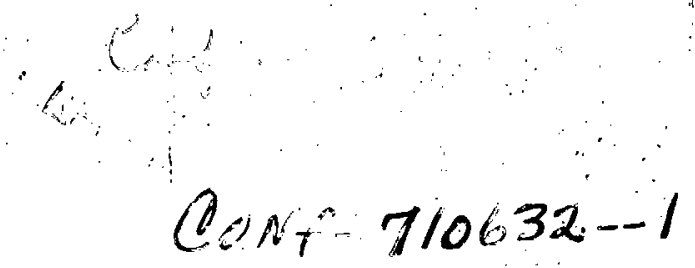

\title{
ON THE STRUCTURE OF HUMAN SERUM LIPOPROTEINS
}

\author{
Angelo M. Scanu, M.D. \\ Professor of Medicine and Biochemistry. \\ The University of Chicago Pritzker School of Medicine \\ and Argonne Cancer Research Hospital* \\ Chicago, Illinois 60637
}

*Operated by the University of Chicago for the United States Atomic

\section{Energy Commission.}

This report was prepared as an account of work
sponsored by the United States Government. Neither
the United States nor the United States Atomic Energy
Commission, nor any of their employees, nor any of
their contractors, subcontractors, or their employees,
makes any warranty, express or implied, or assumes any
legal liability or responsibility for the accuracy, com-
pleteness or usefulness of any information, apparatus,
product or process disclosed, or represents that its use
would not infringe privately owned rights. 


\section{DISCLAIMER}

This report was prepared as an account of work sponsored by an agency of the United States Government. Neither the United States Government nor any agency Thereof, nor any of their employees, makes any warranty, express or implied, or assumes any legal liability or responsibility for the accuracy, completeness, or usefulness of any information, apparatus, product, or process disclosed, or represents that its use would not infringe privately owned rights. Reference herein to any specific commercial product, process, or service by trade name, trademark, manufacturer, or otherwise does not necessarily constitute or imply its endorsement, recommendation, or favoring by the United States Government or any agency thereof. The views and opinions of authors expressed herein do not necessarily state or reflect those of the United States Government or any agency thereof. 


\section{DISCLAIMER}

Portions of this document may be illegible in electronic image products. Images are produced from the best available original document. 
Introduction

High Density Lipoproteins: General Considerations

Analytical Studies

Lipids of HDL

Proteins of HDL

Carbohydrates of HDL

Structural Approaches

Chemical and Enzymatic Studies

Re-assembly Studies

Physical Studies

Ultracentrifugation

Electron microscopy

Optical rotatory dispersion (ORD) and circular dichroism (CD)

Infrared spectroscopy

Fluorescence spectroscopy

Nuclear magnetic resonance (NMR)

Electron spin resonance (esr) - spin labeling

$\mathrm{X}$-ray techniques

Surface balance studies

Overall View on the Structure of HDL 
Introduction

Serum lipoproteins are referred to as "soluble" lipoproteins to specify their solubility in aqueous media, a property not shared by the so-called "structural" lipoproteins which are components of membrane systems. Being watersoluble, serum lipoproteins are more amenable to isolation and purification, thus providing a useful starting point for a thorough analytical examination of their constituents and for an understanding of how such constituents are held together. Serum lipoproteins are fundamental in lipid transport and a knowledge of the ir structural make-up is indispensable for the understanding of their function. Aside from their biological interest, serum lipoproteins represent a useful model for the study of lipid-protein interactions, and information derived from such studies may permit conclusions of general interest.

The present analysis of the structural studies on serum lipoproteins will be limited to the high density class since comparatively more extensive information is available on its physical and chemical properties, and significant contributions have recently been made in the understanding of its structure (see ref, 1 for a recent review).

\section{High Density Lipoproteins: General Considerations}

High density lipoproteins, $\mathrm{HDL}{ }^{\dagger}$ represent a class of lipoprote ins of high hydrated density commonly separated by ultracentrifugation in media of density between 1.063 and $1.21 \mathrm{~g} / \mathrm{ml}$. Two major HDL subclasses have been recognized, $\mathrm{HDL}_{2}$ and $\mathrm{HDL}_{3}$, each with identical but proportionally different prote in and lipid constituents, a fact that appears to account for their distinct molecular weight, 
density, and dimensions (Table 1). HDL , a lipoprote in of inconstant occurrence is probably not a true HDL ${ }^{2}$ and will not be considered in this discussion.

\section{Analytical Studies}

\section{Lipids of HDL}

Phosphatidyl choline and cholesterol esters are the most abundant species, representing about 38 and 27 per cent respectively of the total lipid mass in both $\mathrm{HDL}_{2}$ and $\mathrm{HDL}_{3}$. Among the other phospholipids, sphingomyelin represents about $7 \%$ of the total lipids, whereas lesser amounts of lysophosphatidylcholine, phosphatidylserine, phosphatidylethanolamine and phosphatidylinositol are present. ${ }^{2,3}$ Of the non-P-containing lipids, free cholesterol, triglycerides, diglycerides and monoglycerides have the following distribution. (\% total lipids): $9.0,5.6,0.8$ and 1.2 with some slight variations between $\mathrm{HDL}_{2}$ and $\mathrm{HDL}_{3}$. In all lipid classes the most predominant unsaturated fatty acids are oleic and linoleic ${ }_{\beta}$ whereas palmitic is most important among the saturated alkyl chains. ${ }^{4,5}$ Thus, the lipid moiety of the HDL class is highly heterogeneous for reasons that are as yet undetermined. Proteins of HDL

Many of the analytical studies on HDL proteins have been made possible by the development of a delipidation method, ${ }^{6}$ or modifications thereof, ${ }^{7-9}$ which was initially designed for the removal of lipids from whole serum. ${ }^{10}$ in the technique described by Scanu et al. $i^{7,8}$ the HDL prote in is precipitated by a mixture of 3:2 (v:v) ethanol-ethyl ether at low temperature, yielding a product that contains less than $1 \%$ lipid, and is soluble in aqueous buffers (see, for review, refs. 1 and 11 ). Prote in recoveries are of the order of $90-95 \%$. This incomplete recovery is related to the fact that a portion of the apo HDL, fraction V, (see below) is transferred into 
the organic phase during delipidation as a consequence of its solubility in aqueous ethanol. ${ }^{13}$ Precipitation of this ethanol-soluble fraction is achieved by increasing the ethyl ether:ethanol ratio of the extracting mixture.

Both apo $\mathrm{HDL}_{2}$ and apo $\mathrm{HDL}_{3}$ dissolve in aqueous buffers of low ionic strength to form soluble aggregates readily detectable in the analytical ultracentrifuge. In media of a higher ionic strength and by appropriate $\mathrm{pH}$ adjustments, apo $\mathrm{HDL}_{2}$ or apo $\mathrm{HDL}_{3}$ can be directed into reversible patterns of aggregation of the type: solution $\rightleftharpoons$ fiber $\rightleftharpoons$ gel. ${ }^{13}$ By electron microscopy the isotropic gels were found made of fibrous-like elements of different lengths and orientation and also variable in thickness. Such patterns of aggregation were either prevented or: reversed by lipids, especially by phospholipids: Dissociation of the aggregates into more discrete units was achieved in urea, guanidine hydrochloride; anionic and nonionic detergents, ${ }^{7,8}$ or after chemical modifications such as succinylation. ${ }^{14}$

Such solubility studies provided the basis for development of techniques of isolation of the various polypeptide subunits of apo HDL. Their heterogeneity has been clearly documented by studies of Shore and Shore ${ }_{0}^{15 m-17}$ Scanu et al. ${ }^{18}$ and Rudman et al. ${ }^{19}$ Two major polypeptide classes have been isolated by either DEAE cellulose chromatography or gel filtration (Table 2) with resulting products similar, though not identical, from laboratory to laboratory. Since methods of isolation have not been standardized and the products have not been exhaustively defined, the terminology employed in this article will be that followed in this laboratory. By using the procedure of Scanu et al. ${ }^{18}$ a third minor class of polypeptides, fraction $V$, whose properties are distinctly different from those of fractions III and IV, can be separated. Recent work indicates that each of the three fractions, III, 
$\mathrm{N}$ and $\mathrm{V}$, obtained by gel filtration can be further fractionated into chemically distinct but closely related sub-classes by DEAE cellulose chromatography and isoelectric focusing (Scanu, et al., unpublished). Such polymorphic forms, now in the process of thorough characterization, have also been found in HDL preparations from single donors. Since their structural significance has nof yet been established, the following discussion will refer to components III, N and $V$ as a whole. A more thorough discussion on the properties of the polypeptides of apo HDL is presented elsewhere. ${ }^{2}$

Carbohydrates of HDL

Apo HDL contains 3-4\% carbohydrates, which are attached to the protein presumably by co-valent linkage. No carbohydrates have been found in the lipid moiety. Thus, apo HDL should be considered a glycoprotein. Like other plasma glycoproteins if contains hexose amine, fructose, galactose, mannose and sialic acid." Studies from this laboratory have shown that sialic acid is terminal whereas glucosamine is attached to asparagine by glycosidic linkage (unpublished). Studies on the carbohydrate composition of the various polypeptides of apo HDL are now in progress. The structural significance of the carbohydrates in HDL is totally unclear.

\section{Structural Approaches}

Chemical and Enzymatic Studies

These studies, particularly designed to assess the surface topology of the various functional groups of HDL, have been surprisingly limited. Succinylation of $\mathrm{HDL}_{3}$ or $\mathrm{HDL}_{3}$ has shown that more than $90 \%$ of the free amino groups in the lysine residues are readily accessible to succinic anhydride, thus suggesting the ir surface 
location. ${ }^{14}$ It is particularly interesting that, in spite of this chemical modification, $\mathrm{HDL}_{2}$ or $\mathrm{HDL}_{3}$ retained the same chemical, ultracentrifugal and morphological properties as the non-treated products except for the electrophoretic mobility influenced by the changes in surface charge. The surface localization of a large percentage of the HDL protein has been postulated by Camejo ${ }^{20}$ on the basis of the action of trypsin on intact HDL. However, the kinetics of the reaction were not rigorously followed, the purity of the enzyme was not assessed, and no information was given on the structural stability of the lipoprotein during proteolysis. Ashworth and Green 21 have shown that digestion by either trypsin or chymotrypsin increases the amount of neutral lipids extracted by ethyl ether and also causes release of phospholipids that are not extracted from native HDL by this solvent. A more direct proof for the marked structural effects of trypsin on HDL has been recently obtained in this laboratory (Wisdom and Scanu, unpublished). Upon enzymatic attack, HDL becomes markedly turbid and loses its characteristic flotational properties, a structural alteration that could in part account for the observed complex enzyme kinetics.

Phospholipase D was shown to liberate $88 \%$ of the choline present in HDL. ${ }^{2 I}$ Similarly, phospholipase A attacks more than $95 \%$ of the choline and ethanolamine phospholipids. ${ }^{20}$ It is interesting to note that phospholipase A digested-HDL exhibits essentially the same hydrodynamic behavior as the untreated lipoprotein; Camejo interprets this as meaning that lysophosphatides and fatty acids produced by the enzyme remain fixed at their ordinary sites.

Taken together, the above results do not permit firm conclusions on $H D L$ 
structure. Although they indicare that both protein and phospholipids are at the surface of HDL, they do not specify the quantitative aspects and topology of their participation. More studies are obviously needed in this important area. Re-assembly Studies

Since it was first demonstrated that the prote in moiety of HDL can be isolated in water-soluble form, numerous attempts have been made to restore the lipid complement of this material either partially or totally. The inherent difficulty of this type of experimentation is that proteins and lipids are not soluble in the same solvent system and methods must be devised to overcome this difficulty. Early work from this laboratory showed that radioactively-labeled apo HDL, with either mixed "in vitro" with whole serum or injected intravenously into the animal, acquired sufficient lipids to float in the density limits of HDL. $22-24$. The reaction was essentially specific for HDL, occurred rapidly, produced no detectable intermediates and yielded a labeled HDL indistinguishable from the native lipoprotein. ${ }^{2 a}$ Although such studies provided evidence for the avidity of apo HDL for lipids and for its capacity to recognize its own lipoprote in complex, no information was obtained on the mechanism of re-lipidation. In subsequent studies, apo. HDL was shown to form complexes with sonicated aqueous dispersions of phosphatidylcholine, phosphatidylethanolamine and sphingomyel in isolated from HDL. The better characterized apo HDL-phosphatidylcholine complex could be isolated by ultrocentrifugal flotation at $d i .24$ had a protein:lipid ratio in the same range as that of native HDL and, like the latter, was stable in high salt media. This observation and the fact that apo HDL retained binding property for phospholipids 
after acetylation, succinylation and treatment with neuraminidase were taken to support the predominant non-polar nature of the protein-lipid interactions. ${ }^{25}$ Complex formation was also shown to occur between apo HDL and aqueous dispersions of lecithin plus free cholesterol but not with cholesterol alone. ${ }^{20}$ Essentially similar results were obtained by Sodhi and Gould ${ }^{9}$ with a biphasic system, water:petroleum ether. From the above studies it was concluded that the combination of apo HDL with phospholipids is the primary step in the formation of HDL. This concept has received support from recent studies by Camejo et al. ${ }^{27}$ More information on these partially reconstituted complexes will be given in the following sections.

Under the experimental conditions followed in the above studies no significant incorporation of cholesterol esters or triglycerides in either presence or absence of phospholipids was demonstrated. Recently, incorporation of cholesterol esters into a water-soluble complex resembling native HDL in properties, was achieved by sonicating this lipid with apo HDL and phospholipids under appropriate conditions of vessel geometry, $\mathrm{pH}$ and ionic strength of the medium, temperature, energy output and time. 28,29 Both apo HDL and phospholipids appeared to be necessary for the process since no direct interaction could be demonstrated between apo HDL or cholesterol esters or phospholipids and cholesterol esters. The temperature promoting total HDL re-assembly $\left(40^{\circ} \mathrm{C}\right)$ was found to correspond to the "melting" temperature of the mixture of cholesterol esters alkyl chains in HDL, as assessed by $x$-ray diffraction techniques. ${ }^{30}$. In such experiments, the previously observed lack of interaction between cholesterol esters and phospholipids was confirmed. 
It was also shown that either the native or the totally reconstituted product is in a "fluid" or "unordered" conformation as determined by the presence of the $54.5 \AA^{-1}$ band in the $x$-ray patterns.

In the course of the studies on the re-assembly of apo HDL, some information on the affinity for lipids of the various apo HDL subunit was also obtained. When sonicated mixtures of apo HDL and HDL lipids were fractionated by sequential flotation, three fractions were obtained: two floating at $d<1.063$ and $d 1.063-$ $1.21 \mathrm{~g} / \mathrm{ml}$, respectively and the thord one sedimenting at $d>1.21 \mathrm{gm} / \mathrm{ml}$. The lightest complex contained $V$ and $V$ polypeptides; the 1.21 top contained fractions III and IV, while the 1.21 bottom was composed exclusively of fraction III. When each polypeptide class was studied individually, it was shown that both III and IV when sonicated in the presence of whole HDL lipids will form HDL species that are distinct from each other from the physico-chemical standpoint. The HDL with fraction III more closely resembles the native HDL. When polypeptides of class $V$ were studied in a similar manner, they associated preferentially with neutral lipids to form complexes floating in the low density range. ${ }^{29}$ Such observations have recently been confirmed by Forte and Nichols. ${ }^{31}$

Thus, these techniques permit a graded re-lipidation of apo HDL up to a complex very similar to the native one. More details on the physical properties of the partially and totally reconstituted complexes will be given in the following section.

Physical Studies

Ultracentrifugation. --Both preparative and analytical ultracentrifugation 
have been extensively applied to the study of plasma lipoproteins. It is, in fact, customary to define plasma lipoproteins in terms of their flotational rate, a parameter which, when used alone, is not sufficient to identify a distinct lipoprotein species and its ho:mogeneity. This is due to the fact that there is a wide $S_{f}$ distribution within lipoprotein classes, a reflection of variations in size and density. In the case of HDL, two major sub-classes of lipoproteins have been identified by Lindgren and Nichols. ${ }^{32}$ On the other hand, it has been suggested that the high density lipoproteins represent a single lipoprotein class varying in composition throughout a density continuum. The view that $\mathrm{HDL}_{2}$ and $\mathrm{HDL}_{3}$ are simply ultracentrifugal artifacts ${ }^{33}$ has been challenged. ${ }^{2}$ - Thus, the exact structural and metabolic relationship between these two sub-classes remains to be established.

Ultracentrifugation with particular regard to flotational techniques has been valuable in the re-assembly study from both the preparative and analytical standpoints. ${ }^{9,25039}$ With regard to the total re-assembly work, it has been possible to show that the major portion of the product has flotational properties of $\mathrm{HDL}_{3}{ }^{29}$ and that other lipoprotein species are formed. In a similar fashion, information has been obtained on the effect of sonication on high density lipoproteins as well as the effect of various agents affecting HDL stability. When coupled with other physical parameters, the information should prove valuable in the understanding of HDL structure.

Electron microscopy.--The various serum lipoproteins can be visualized in the electron microscope after negative staining by sodium phosphotungstate. $29,34,35$ 
When applied to HDL particles they were found to have an average diameter of $86 \AA$ (range 74-98 $)$, whereas the two sub-classes, $\mathrm{HDL}_{2}$ and $\mathrm{HDL}_{3}$ had an average diameter of $95 \AA$ and $65 \AA$, respectively. ${ }^{34}$ By invoking various assumptions these authors calculated molecular weights for these fractions in reasonable agreement with the values obtained in the ultracentrifuge. ${ }^{2}$. An analysis of totally re-assembled materials from apo HDL showed spherical units, similar in form to those of native $H D L$ but with a layer size (110 $\AA$ in diameter) that was comparable to that of native HDL after sonication. ${ }^{29}$ Materials reconstituted from III and IV differed in size, average diameters being 120 and $180 \AA$, respectively. Particularly striking was the size heterogeneity of the particles reconstituted from $\mathrm{N}_{;}$this is in keeping with the results obtained by the analytical ultracentrifuge.

Forte et al.$^{34,38}$ drew attention to the presence of a substructure in HDL. They interpreted the morphological appearance as showing an electron-dense central core surrounded, in a ring-like fashion, by 4-5 subunits each with a diameter of $35-50 \AA$. The nature of the postulated subunits (protein, lipid, lipoproteins?) was not determined. Recently, Forte et al. ${ }^{36-}$ published the results of studies on HDL obtained from patients with familial lecithin-cholesterol acyltransferase deficiency, a disease associated with low levels of plasma cholesterol esters. The majority of the particles appeared by negative staining with phosphotungstate to be disc-shaped structures with a major diameter of 150-200 $\AA$ and a thickness of 50-55 $\AA$, and had a tendency to form stacks of variable length. In such stacks, discs were separated from each other by a PTA-containing hydrophilic region of $10 \AA$ thickness. The authors noted that the $50-55 \AA$ periodicity in the stacked discs 
was similar to that seen in the bilayer structures formed by negatively stained phospholipids or phospholipid-cholesterol dispersions 37,38 or phospholipids, cholesterol and bile acids. Since the abnormal HDL particles contained no cholesterol esters, their tendency to form disc-like structures was attributed to the absence of the non-polar "core" of lipids, a conclusion in keeping with the results of the re-assembly studies by Scanu et al.,$^{29}$ and those by the authors ${ }^{36}$ who show that disc-like structures made up of apo HDL subunits, phospholipids and free cholesterol can be transformed into spherical structures, characteristic of normal HDL, by addition of cholesterol esters. It is of interest to point out that disc-like structures have been seen by electron microscopy in the abnormal low density lipoproteins of cholestasis, particles characterized by nearly equimolar amounts of phospholipids and free cholesterol, a small amount of protein, but no cholesterol esters. $^{\text {a5 }}$

Optical rotatory dispersion (ORD) and circular dichroism (CD).--The first ORD studies by Scanu ${ }^{40}$ on HDL and apo HDL showed that both products exhibited a spectrum characterized by a negative maximum at $233 \mathrm{~nm}$, a crossover point at $225 \mathrm{~nm}$ and a positive maximum at $198 \mathrm{~nm}$. From the rotational amplitudes it was estimated that the prote in of HDL had a relatively high a-helical content, $60-70 \%$, which fell to about $55-65 \%$ following delipidation. An interesting concept that derived from these studies was the partial shielding effect of the lipid on the denaturation of apo $\mathrm{HDL}_{2}$ by a number of perturbing agents. Since these early studies, more extensive work has been carried out on HDL and its derivatives by $C D$. The CD studies confirmed the high helical content in both HDL and apo HDL, 
and in addition detected significant spectral differences between the two products in terms of relative ratios of the ellipticity minima of the 222 and $208 \mathrm{~nm}$ bands. ${ }^{41}$ Temperature studies showed again the protective effect of lipids on the conformation of apo HDL. The temperature-induced conformational transitions were found to be reversible although the course of renaturation was slower in the case of apo $\mathrm{HDL}_{3} \cdot \cdot^{26}$ An analysis of the two major apo HDL subunits by Gotto and Shore ${ }^{42}$ indicated a greater $\alpha$-helical content in $R$-thr than in $R$-gln. A similar, though less marked, difference was noted by Scanu ${ }^{43}$ between fractions III and IV. In the latter studies it was also shown that reduction and carboxymethylation affected only the $C D$ spectrum of $N$. This was related to the presence of cystine in this fraction, an interpretation supported by the finding that the spectrum of reduced fraction IV could be restored to normal upon re-oxidation by air.

When applied to the re-assembled products, the CD studies showed that addition of phospholipids or phospholipids and free cholesterol did not change significantly the spectrum of apo HDL: Only the totally reconstituted products, i.e., those containing cholesterol esters, exhibited a spectrum that was indistinguishable from that of native HDL. ${ }^{29}$ Thus, neutral lipids appear to have an important structural role in HDL, a conclusion in keeping with the results of the studies reported in the preceding sections.

Infrared spectroscopy.--Infrared spectroscopy has seen only a limited application to the study of the structure of HDL. The first studies reported by Scanu 44 in native HDL indicated a position for the amide I and amide II bands at $1650 \mathrm{~cm}^{-1}$. and $1540 \mathrm{~cm}^{-1}$, respectively, as expected for a protein with predominance of an 
a-helix. For apo HDL and the reconstituted apo HDL-phospholipids complex, the amide I and amide II bands occurred at slightly lower frequencies, namely at $1645 \mathrm{~cm}^{-1}$ and $1535 \mathrm{~cm}^{-1}$. In more recent studies Gotto and Kon 45 found that the amide I infrared band for the native $H D L$ and apo $H D L$ in $D_{2} O$ occurred at a significantly lower frequency $\left(1637-1640 \mathrm{~cm}^{-1}\right)$ than that found by Scanu for HDL. The authors could not explain this discrepancy, although they pointed out that a similar result was obtained with the $\alpha$-helical polypeptide, polyglycine II. Fluorescence spectroscopy.--In spite of the extensive use of fluorescence probes especially in the study of apolar interactions, only limited information is available on the application of such techniques to the study of the structure of serum lipoproteins. Hart ef al ${ }^{46^{*}}$ interpreted their fluorescence data to mean that the aromatic amino acids of $\mathrm{HDL}_{2}$ are located in a relatively polar environment, probably at or near the solvent interface, a concept supported by the finding that the emission maximum of the intrinsic fluorescence was not significantly affected by delipidation. Intensity and emission wavelength studies also showed that the $\mathrm{HDL}_{2}$ lipids partially protected the protein from undergoing thermal or urea-induced conformational changes. Studies with the fluorescence probe ANS (1-anilino-8naphthalene sulphonate) suggested that three apolar binding sites are present in apo $\mathrm{HDL}_{2}$. It is interesting that when similar studies were carried out with native $\mathrm{HDL}_{2}$, there was a 200-fold increase of the fluorescent intensity, which again suggests the existence of extensive apolar regions in $\mathrm{HDL}_{2}$. Because of the versatility of the fluorescence spectroscopy technique and the availability of a number of reporter molecules it is expected that this technique will provide important structural information on HDL. 
Nuclear magnetic resonance (NMR). --From the theoretical standpoint, the NMR technique appears well suited for the investigation of lipid:protein interactions in HDL and for providing significant information complementary to that obtained by other physical methods. However, we should be aware of the fact that HDL has several chemical groupings which may not be readily resolved; thus causing difficulties in the interpretation of the chemical shifts.

The $220 \mathrm{MHz}$ spectrum of $\mathrm{HDL}_{2}$ in $\mathrm{D}_{2} \mathrm{O}$ reported by Chapman et al. ${ }^{47}$ showed a number of signals only a few of which could be assigned without ambiguity both in terms of molecular types (phospholipids, protein, cholesterol esters) and sub-molecular grouping (i.e., choline methyl groups, aromatic amino acids). Although the results were of interest, such spectra provided little information on the lipid:lipid or protein:lipid interactions in HDL. By line-broadening criteria there was no clear indication for apolar interactions. In fact, based on the sharpness of the observed signals, one may derive the conclusion that lipid alkyl chains, the polar choline groups and at least some of the amino acids are quite unrestrained in motion, which indicates that such groupings are not involved in apolar interactions. Steim et al. ${ }^{48}$ who obtained spectra at $60 \mathrm{MHz}$ showing the same general although less resolved features of the $200 \mathrm{MHz}$ studies, interpreted the ir results to support a "micellar" model for HDL structure, thus minimizing the significance of apolar interactions. The authors, however, have recognized the lack of quantification inherent in NMR techniques and the possibility that a certain percentage of the protein and lipid protons which are constrained in their motion may be undetected. When the studies were extended to apo $\mathrm{HDL}_{2},{ }^{47}$ the $220 \mathrm{MHz}$ spectra showed a 
relatively poor definition of the apoprotein signals attributable to either its state of aggregation or its conformation. In the presence of urea, or by raising the temperature, the spectra improved in resolution probably due to the unfolding of the polypeptide chains. This allowed for the identification of a region $(2-3 \tau)$ due to the aromatic amino acids and distinct from the sharp signals of the phospholipid choline groups $(6-7 \tau)$, angular methyl groups of cholesterol $(9 \tau)$ and cholesterol esters $(9.3 \tau)$. It may be added that when similar perturbation conditions were applied to whole $\mathrm{HDL}_{2}$ the induced spectral changes were less significant, pointing out again the protective effect of lipids on the conformation of proteins.

The partially reconstituted $\mathrm{HDL}$ obtained by combining apo $H D L_{2}$ and lecithin gave spectra quite similar in overall appearance, indicating that the individual proton grouping had similar environments and molecular mobility both in the native and reconstituted lipoprotein, although differences were noted at given temperatures. ${ }^{47}$ When the studies were extended to the totally reconstituted products a closer similarity with $H D L_{2}$ was noted in terms of overall appearance, line width and resolution, temperature response and appearance of cholesterol, ester peaks (R. B. Leslie and A. Scanu, unpublished).

No systematic work has yet been reported on the protein subunits of apo HDL or on their products of reconstitution. The analysis of such products may provide results of clearer interpretation and of obvious significance to the problem of HDL structure.

Electron spin resonance (esr) - spin labeling.--One of the drawbacks of the NMR technique is its limited sensitivity, which demands either high sample 
concentrations or a particularly designed spectrometer. The esr technique has no such limitations, although it requires the presence of paramagnetic groups usually not present in biological systems. This problem has been, to a large extent, overcome by the work of McConnell et al..$^{49}$ who showed that special stable free radicals, better known as spin labels, can be attached to molecules or molecular systems either co-valently or non co-valently. Since this demonstration, the spin label method has been very widely applied to a number of biological problems with results not always unequivocal.

The first application of the spin label technique to serum lipoproteins was reported by Gotto and Kon ${ }^{\text {so }}$ using the nitroxide radical $\mathrm{N}-(1-0 x y \mid-2,2,6,6-$ tetramethyl-4-piperidinyl)isothiocyanate which is believed to label the 6 -amino groups of lysine. As judged by immunochemical and circular dichroic measurements, no significant structural changes occurred in the molecule following labeling. The spectrum exhibited a broad and a narrow component, the former reflecting strong immobilization of the radical, while the latter was attributed largely to unrestricted motion of the probe. The strong immobilized component was attributed to proteinlipid interaction since it would be, to a great extent, replaced by a narrow signal upon delipidation. ${ }^{45,50,51}$ Gotto et al. ${ }^{51}$ also observed that the partially reconstituted complex made by combining spin-labeled apo HDL and phospholipids gave an esr spectrum similar to that of the apoprotein alone, which is in keeping with the previously discussed results by circular dichroism and nuclear magnetic resonance. Recent studies from this laboratory ${ }^{52,53}$ using a mixed anhydride label have given results in general agreement with those of Gotto et al. ${ }^{52}$ In addition, the results 
described for apo HDL were found to apply to its subunits III and IV, which exhibited a similar $\mathrm{pH}$ and temperature behavior. It was significant that the spectrum of totally reconstituted $H D L$ was strikingly similar to that of native $H D L_{a}$, pointing again to the importance of cholesterol esters in the HDL structure. From the esr standpoint a close similarity was observed between complexes made by combining apo $\mathrm{HDL}_{2}$ or its subunits III and $N$ with either whole $\mathrm{HDL}_{2}$ lipids or mixtures of egg lecithin and cholesterol oleate. Aside from this structural information, the spin label methods failed to provide molecular details on the nature of the protein-lipid interactions in HDL. It is hoped that such information will be derived from the use of more specific labels for each of the lipoprotein constituents, an experimental approach that should be favored by the development of re-assembly techniques discussed in one of the previous sections.

$\underline{X \text {-ray techniques. }--T h e ~ t e c h n i q u e ~ o f ~ s m a l l ~ a n g l e ~} x$-ray scattering provides a method for determining the size, shape, molecular weight or other associated parameters of macromolecules in ideally monodisperse dilute solutions. The interpretation of a scattering curve may pose complex problems that may be minimized if ancillary chemical and physical parameters of the sample under study are known. A small angle $x$-ray analysis of human serum $\mathrm{HDL}_{2}$ was recently carried out by Atkinson et al.$^{54}$ using a slit collimated $x$-ray beam. The overall features of the scattering curve, particularly the well-defined secondary scattering maxima, indicated that $\mathrm{HDL}_{2}$ is probably a highly symmetrical particle. The position and infensities of the secondary maxima did not coincide with those expected for a spherical particle of uniform electron density. According to Atkinson et al. ${ }^{54}$ the differences 
in position and intensity could be explained by a long range structural organization within the particle which results in a radial electron density distribution. This would be characterized by a relatively electron deficient, i.e., hydrocarbons central core surrounded by an electron rich outer shell containing the protein and lipid head group. This is consistent with the suggestion, summarized in the previous sections, that the protein subunits and polar moieties of the phospholipids are located at the surface of the HDL particle. The radius of gytation obtained from the "smeared" scattering curve was $44 \AA$, and from this a value of $108 \AA$ for the diameter of the particle could be calculated, which is in agreement with the figure obtained by electron microscopy. Work was also carried out in the totally re-assembled complex. The results, although preliminary, indicate great similarities between these complexes and the native ones. Clearly the small angle x-ray technique is applicable to a system as complex as human serum $\mathrm{HDL}_{2}$. Continuing work on this area is likely to prove highly fruitful, especially if systematically applied to the various HDL derivatives.

Surface bálance studies. --Monolayer techniques have had only a limited application to the study of protein-lipid interactions in HDL. The first studies by Camejo et al. ${ }^{55}$ were carried out with rat serum apo HDL, using lipid films with both low $(2 \mathrm{dyne} / \mathrm{cm})$ and high $(15 \mathrm{dyne} / \mathrm{cm})$ initial pressure. At 2 dyne $/ \mathrm{cm}$, the extent of film penetration and the final equilibrium surface pressure were significantly larger with apo HDL than with plasma albumin. Using films made of the individual phospholipid classes, the degree of interaction was found to be phosphatidylethanolamine $>$ phosphatidylcholine $>$ lysophosphatidylcholine $>$ sphingomyelin. 
Using films at the initial pressure of $15 \mathrm{dyne} / \mathrm{cm}$, the order of interaction as judged by the final equilibrium surface pressure was: phosphatidylethanolamine >phosphatidylcholine > sphingomyelin >lipolecithin with monolayers made of total HDL lipids. The final equilibrium surface pressure and rate of protein penetration obtained with apo HDL were from two to four-fold as large as was obtained with albumin. In addition, it was found that apo HDL is highly surface-active and interacts with lipid monolayers even at its collagen pressure (15 dyne $/ \mathrm{cm})$.

According to Camejo 20 human serum apo HDL has a surface behavior similar to that of rat apo HDL. Using various assumptions and the information derived from the monolayer experiments, Camejo interpreted his results to mean that the polypeptides in HDL are not fully extended but in a compact organization, which is compatible with the estimates of $\alpha$-helical content from ORD and CD studies. Such a concept is in obvious need of experimental testing, possibly by $x$-ray crystallographic analysis.

More recently, Camejo et al. ${ }^{27}$ have examined polypeptide fractions from human apo HDL by preparative polyacrylamide gel electrophoresis. Fractions b and $c$, unfortunately not defined chemically, were those that were surface-active and had final surface pressures similar to that of whole apo HDL. Such fractions were shown to penetrate lipid films at low $(2$ dyne $/ \mathrm{cm})$ and high $(16$ dyne $/ \mathrm{cm})$ initial pressures. This property was not shared by the other minor components of apo HDL. Surface balance studies utilizing polypeptides with well-defined physical and chemical properties are awaited with great interest. 
Overall View on the Structure of HDL

In spite of the relatively large body of experimental data which have been obtained by chemical, enzymatic and physical methods, the formulation of a structural model for HDL is still in need of speculative thoughts. In terms of the protein moiety, the water solubility of HDL, the chemical and enzymatic accessibility data, the information derived from NMR and spin labeling techniques together with the surface balance studies, all appear to suggest that a large portion of apo $\mathrm{HDL}$ is located at the surface as a highly organized structure (see $O R D, C D$ and IR data). The structural role played by each of the major polypeptide subunits remains undetermined and in this context one cannot rule out the possibility that the materials isolated in the ultracentrifuge as $\mathrm{HDL}_{2}$ or $\mathrm{HDL}_{3}$ may in fact be composed of two isodense species with distinct polypeptide chains. This concept receives support from the re-assembly work but it is in need of a more direct documentation from studies on native HDL particles. Another totally unresolved problem is that of the structural role of the polysaccharides in HDL, a moiety that has not yet received extensive experimental attention.

In terms of phospholipids, the surface location of the choline polar groups is suggested by the NMR studies, their accessibility to phospholipase $A$, and also by their known tendency to reside at a polar/nonpolar interface. In turn, it is more likely that the hydrocarbon chains are located in the interior of the molecule, where they form an extensive non-polar region. This is compatible with the fluorescence studies with ANS, the NMR and the $x$-ray data.

The structural role of cholesterol esters in HDL has become apparent only 
recently chiefly through the re-assembly studies and the analysis of lipoproteins genetically deprived of the ir cholesterol esters complement. Such studies have indicated that without cholesterol esters, HDL particles do not exhibit the morphological appearance, spectroscopic properties and thermal stability of the native products, properties which can be restored by the addition of cholesterol esters. Their non-polar nature, together with thermodynamic considerations, leads to the assignment of cholesterol esters as "core" molecules around which a polar region consisting of polypeptides, phospholipids and free cholesterol is organized. Such molecules would appear to have considerable molecular motion as indicated by the signals from $C_{18}$ and $C_{19}$ angular methyl groups in the NMR spectro at high temperature and also by the very recent $x$-ray diffraction data.

In trying to piece together the information so far obtained in terms of HDL structure, a major drawback is our present lack of understanding of the mode of interactions of the various HDL components and of the energetics involved in such interactions. It may be stated that a structure with the polar groups totally or predominantly "outside" and non-polar groups "inside" is thermodynamically best suited for an aqueous environment and this may well apply to HDL. In support of such a concept are many of the data discussed in the previous sections, as well as the recent $x$-ray scattering data by Atkinson et al. ${ }^{54}$ which indicate that HDL has an organized symmetrical structure generating a radial distribution of electron. density, with a relatively electron-poor central (non-polar) region, and a relatively electron-rich (polar) outer region. It should be mentioned that a subunit structure for. HDL has been proposed by Gotto ${ }^{56}$ based primarily on the electron microscopy 
data by Forte et al. ${ }^{37}$ Each of the 4 to 6 subunits would have its own complement of protein, phospholipids and neutral lipids packed into a structure of the dimensions of HDL. The likelihood of such a model has been questioned by Leslie ${ }^{57}$ on the basis of scale model analysis, and appears also to be incompatible with the current $x$-ray observations by Atkinson et al. ${ }^{54}$ Only future studies are likely to reconcile these views and provide a better insight into the internal organization of HDL. To this end, an optimistic attitude is justified by the rapid conceptual and technological advances in the area of lipid-protein interactions in general, and by the possibility of systematically analyzing the mechanisms underlying the re-assembly of HDL from : its individual constituents. 


\section{References}

1. SCANU, A. 1971. Human plasma high density lipoproteins. Biochem. J., in press:

2. SCANU, A., \& J. L. GRANDA. 1966. Effects of ultracentrifugation on the human serum high density $(1.063<\rho<1.21 \mathrm{~g} / \mathrm{ml})$ lipoprotein. Biochemistry $\underline{5}: 446$.

3. SKIPSKI, V. P. , M. BARCLAY, R. K. BARCLAY, V. A. FITZER, J. J. GOOD; \&

F. M. ARCHIBALD. 1967. Lipid composition of human serum lipoproteins. Biochem. J. 104: 340 .

4. NICHOLS, A. V., C. S. REHNBORG, F. T. LINDGREN, \& P. D. WILLS. 1962. Effects of oil ingestion on lipoprotein fatty acids in man. J. Lipid Res. 3: 320.

5. GOODMAN, DeW. S., \& T. SHIRATORI. 1964. Fatty acid composition of human plasma lipoprotein fractions. J. Lipid Res. 5: 307.

6. SCANU, A., L. A. LEWIS, \& M. BUMPUS. 1958. Separation and characterization of the protein moiety of human $\alpha_{1}$-lipoprotein. Arch. Biochem. Biophys. 74: 390 .

7. SCANU, A. 1966. Forms of human serum high density lipoprotein. J. Lipid Res. 7: 295.

8. SHORE, V.., \& B. SHORE. 1967. Some physical and chemical studies on the protein moiety of a high density $(1.125-1.195 \mathrm{~g} / \mathrm{ml})$ lipoprote in fraction of human serum. Biochemistry 6: 1962 .

9. SODHI, H. S., \& R. G. GOULD. 1967. Combination of delipidized high density lipoprotein with lipids. J. Biol. Chem. 242: 1205.

10. SCANU, A., \& N. SCHIANO. 1954. Method of continuous ether extraction of lipids applied to the study of serum lipoproteins. 1. Normal human serum. Rev. Ist. Sierat. Ital. 29: 457.

11. SCANU, A. 1965. Factors affecting lipoprotein metabolism. Adv. Lipid Res. 3 : 63. 
12. SCANU, A., \& C. EDELSTEIN. 1971. Solubility in aqueous solutions of ethanol of the small molecular weight peptides of serum very low-density and high density lipoprote ins. Relevance to a problem of delipidation of plasma lipoprotein. Anal. Biochem., submitted for publication.

13. SCANU, A., \& C. EDELSTEIN. 1971. Reversible directed aggregation of the polypeptides of human serum high density lipoproteins. Abstracts of the 150th Meeting of the American Chemical Society.

14. SCANU, A., W. READER, \& C. EDELSTEIN. 1968. Molecular weight and subunit structure of human serum high density lipoproteins before and after delipidation. Biochim. Biophys. Acta 160: 32.

15. SHORE, B., \& V. SHORE, 1968. Heterogeneity in protein subunits of human serum high density lipoproteins. Biochemistry 7: 2773.

16. SHORE, V., \& B. SHORE. 1968. Some physical and chemical studies in two polypeptide components of high density lipoproteins of human serum. Biochemistry Z: 3396 .

17. SHORE, B., \& V. SHORE. 1969. Isolation and characterization of polypeptides of human serum lipoproteins. Biochemistry 8: 4510.

18. SCANU, A., J. TOTH, C. EDELSTEIN, S. KOGA, \& E. STILLER. 1969. Fractionation of human serum high density lipoproteins in urea solutions. Evidence for polypeptide heterogeneity. Biochemistry $8: 3309$.

19. RUDMAN, R., L. A. GARCIA, \& C. H. HOWARD. 1970. A new method for isolating the non-identical protein subunits of human plasma a-lipoprotein. J. Clin. Invest. 49: 1536 .

20. CAMEJO, G. 1969. The structure of human density lipoprotein: a study of the effect of phospholipase and trypsin on the components and of the behavior of the lipid and prote in moieties at the air-water interface. Biochim. Biophys. Acta 175: 290 . 
21. ASHWORTH, L. A. E., \& C. GREEN. 1963. The action of enzymes on human a-lipoprotein. Biochem. J. 89: 561.

22. SCANU, A., \& W. L. HUGHES. 1960. Recombining capacity towards lipids of the protein moiety of human serum $\alpha$-lipoprotein. J. Biol. Chem. 235: 2876.

23. SCANU, A., \& W. L. HUGHES. 1962. Further characterization of the human serum D 1.063-1.21 $\alpha_{1}$-lipoprotein. J. Clin. Invest. 41: 1681.

24. SCANU, A., \& I. H. PAGE. 1961. Recombination with lipids of the lipid-free prote in from canine serum (d 1.063-1.21, $a_{1}$ ) lipoprotein. J. Lipid Res. 2: 161.

25. SCANU, A. 1967. Binding of human serum high density lipoprotein apoprotein with aqueous dispersions of phospholipids. J. Biol. Chem. 242: 711.

26. SCANU, A. 1969. On the temperature dependence of the conformation of human serum high density lipoproteins. Biochim. Biophys. Acta 181: 268.

27. CAMEJO, G., Z. M. SUAREZ, \& V. MUNOZ. 1970. The apolipoproteins of human plasma high density lipoprotein: a study of their lipid binding capacity and interaction with lipid monolayers. Biochim. Biophys. Acta 218: 155.

28. HIRZ, R., \& A. SCANU: 1970. Re-assembly in vitro of a serum high density lipoprotein. Biochim. Biophys. Acta 207: 364.

29. SCANU, A., E. CUMP, J. TOTH, S. KOGA, E. STILLER, \& L. ALBERS 1970. Degradation and re-assembly of a human serum high density lipoprotein. Evidence for differences in lipid affinity among three classes of polypeptide chains. Biochemistry 9: 1327.

30. SCANU, A., \& A. TARDIEU. 1971. Temperature transitions of mixtures of lipids containing cholesterol esters. Relevance to the problem of structure of serum high density lipoproteins. Biochim. Biophys. Acta 231: 170. 
31. FORTE, G. M., \& A. V. NICHOLS. 1971. Re-assembly of peptides and lipid constituents of serum high density lipoproteins. Abstracts of 15th Meeting of Biophysical Society, p. 125A.

32. LINDGREN, F. T. ; \& A. V. NICHOLS. 1960. Structure and function of human serum lipoproteins. In The Plasma Proteins, F. W. Putnam, Ed. Vol. II, p. 2. Academic Press, New York.

33. LEVY, R. I., \& D. S. FREDRICKSON. 1965. Heterogeneity of plasma high density lipoproteins. J. Clin. Invest. 44: 426.

34. FORTE, G. M. , A. V. NICHOLS, \& R. M. GLAESER. 1968. Electron microscopy of human serum lipoproteins using negative staining. Chem. Phys. Lipids 2: 396.

35. HAMILTON, R. L., R. J. HAVEL L J. P. KANE, A. E. BLAUROCK, \& T. SATA. 1971. Cholestasis: lamellar structure of the abnormal human serum lipoproteins. Science 172: 475 .

36. FORTE, G. M., P. K. NORUM, J. A. GLOMSET, \& A. V. NICHOLS. 1971. Plasma lipoproteins in familial lecithin-cholesterol acyltransferase deficiency: structure of low and high density lipoproteins as revealed by electron microscopy. J. Clin. Invest. 50: 1141 .

37. BANGHAM, A. D., \& R. W. HORNE. 1964. Negative staining of phospholipids and their structural modification by surface-action agents as observed in the electron microscope. J. Mol. Biol. 8: 660 .

38. LUCY, J. A., \& A. M. GLAUERT. 1964. Structure and assembly of macromolecular lipid complexes composed of globular micelles. J. Mol. Biol. 8: 727. 
39. HOWELL, J. I., J. A. LUCY, A. C. PINOLA, \& I. A. D. BOUCHIER. 1970. Macromolecular assemblies of lipids in bile. Biochim. Biophys. Acta 210: 1.

40. SCANU, A. 1965. Studies on the conformafion of human serum high-density lipoproteins, $\mathrm{HDL}_{2}$ and $\mathrm{HDL}_{3}$. Proc. Nat. Acad. Sci., U.S. 54: 1699.

41. SCANU, A., \& R. HRZ. 1968. On the structure of human serum high density lipoprotein: studies by the technique of circular dichroism. Proc. Nat. Acad. Sci., U.S. 59: 890 .

42. GOTTO, A. M., \& B. SHORE. 1969. Conformation of human serum high density lipoprote in and its peptide components. Nature 224: 69.

43. SCANU, A. 1970. The effect of reduction and carboxymethylation on the circular dichroic spectra of two polypeptide classes of serum high density lipoprote ins. Biochim. Biophys. Acta 200: 570.

44. SCANU, A. , \& J. L. GRANDA. 1968. Comparative optical properties of human serum low- and high-density lipoproteins before and after delipidation. Progr. Biochem. Pharmacol. 4: 153.

45. GOTTO, A. M. \& \& H. KON. 1970. Observations on the conformation of human serum high density lipoproteins using infrared spectroscopy, circular dichroism and electron spin resonance. Biochemistry 9: 4276.

46. HART, C. J., R. B. LESLIE, \& A. SCANU. 1970. Fluorescence studies of a high density lipoprotein. Chem. Phys. Lipids 4: 367.

47. CHAPMAN, D., R. B. LESLIE, R. HIRZ, \& A. SCANU. 1969. High-resolution NMR spectra of high density serum lipoproteins. Biochim. Biophys. Acta 176: 524. 
48. STEIM, J. M., O. J. EDNER, \& F. G. BARGOOT. 1968. Structure of human serum lipoprote ins: nuclear magnetic resonance support of a micellar model. Science 162: 909.

49. STONE, T. J., T. BUCKMAN, P. L. NORDIO, \& H. M. MCCONNELL. 1965. Spin-labeled biomolecules. Proc. Nat. Acad. Sci. 54: 1010.

50. GOTTO, A. M., \& H. KON. 1969. Application of electron spin resonance to the study of the structure of human serum lipoproteins. Biochem. Biophys. Res: Comm. 37: 444 .

51. GOTTO, A. M., H. KON, \& M. E. BIRNBAUMER. 1969. Electron spin resonance studies of lipid-protein interactions in human serum lipoproteins. Proc. Nat. Acad. Sci., U. S. 65: 145.

52. BARRATT, M., \& A. SCANU. 1971. Protein-protein and protein-lipid interactions studied by a spin label method. Fed. Proc. 30: 1187Abs.

53. BARRATT, M., R. B. LESLIE, \& A. SCANU. 1971. Protein-protein and proteinlipid interactions in human serum high density lipoprote ins: an analysis in a spin label method. Chem. Phys. Lipids, submitted for publication.

54. ATKINSON, D., G. C. SHIPLEY, \& A. SCANU. 1971. Small angle x-ray scattering studies of human serum high density lipoproteins. In preparation.

55. CAMEJO, G., G. COLACICCO, \& M. M. RAPPORT. 1968. Lipid monolayers: interactions with the apoprotein of high density plasma lipoprotein. J. Lipid Res. 9: 562.

56. GOTTO, A. M. 1969. Recent studies on the structure of human serum low- and high-density lipoproteins. Proc. Nat. Acad. Sci., U.S. 64: 1119. 
57. LESLIE, R. B. 1971. Some physical and physico-chemical approaches to the structure of serum high density lipoproteins. Biochem. J., in press. 


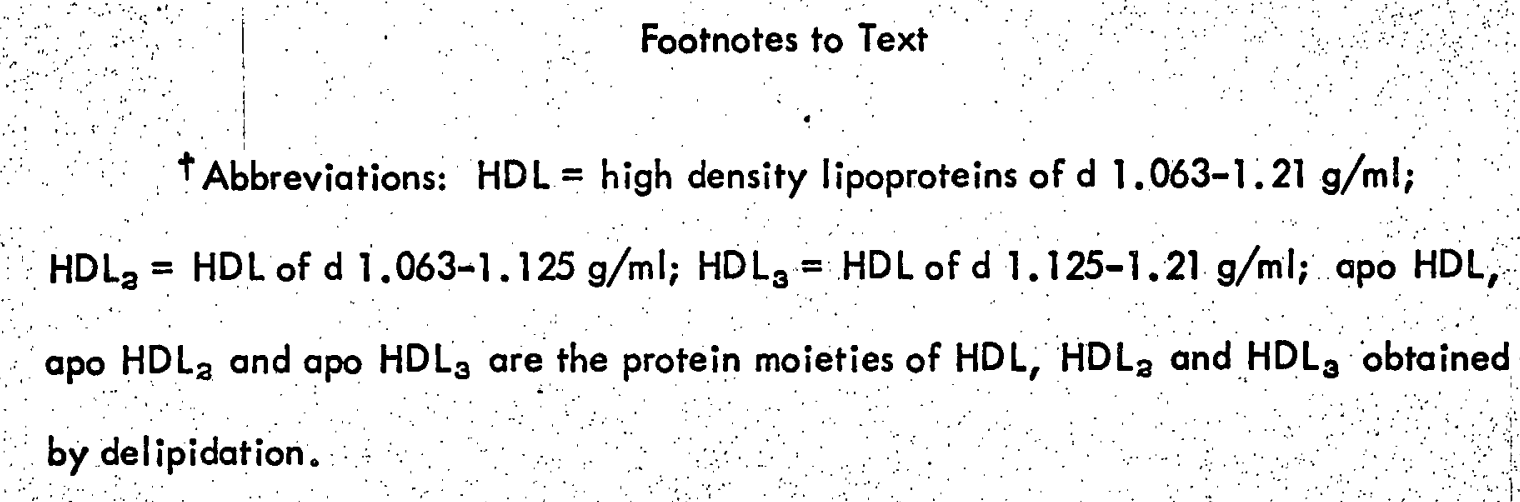


TABLE 1

CHEMICAL COMPOSITION AND SOME OF THE PHYSICAL

PARAMETERS OF HDL $\mathrm{H}_{\mathbf{a}}$ AND HDL

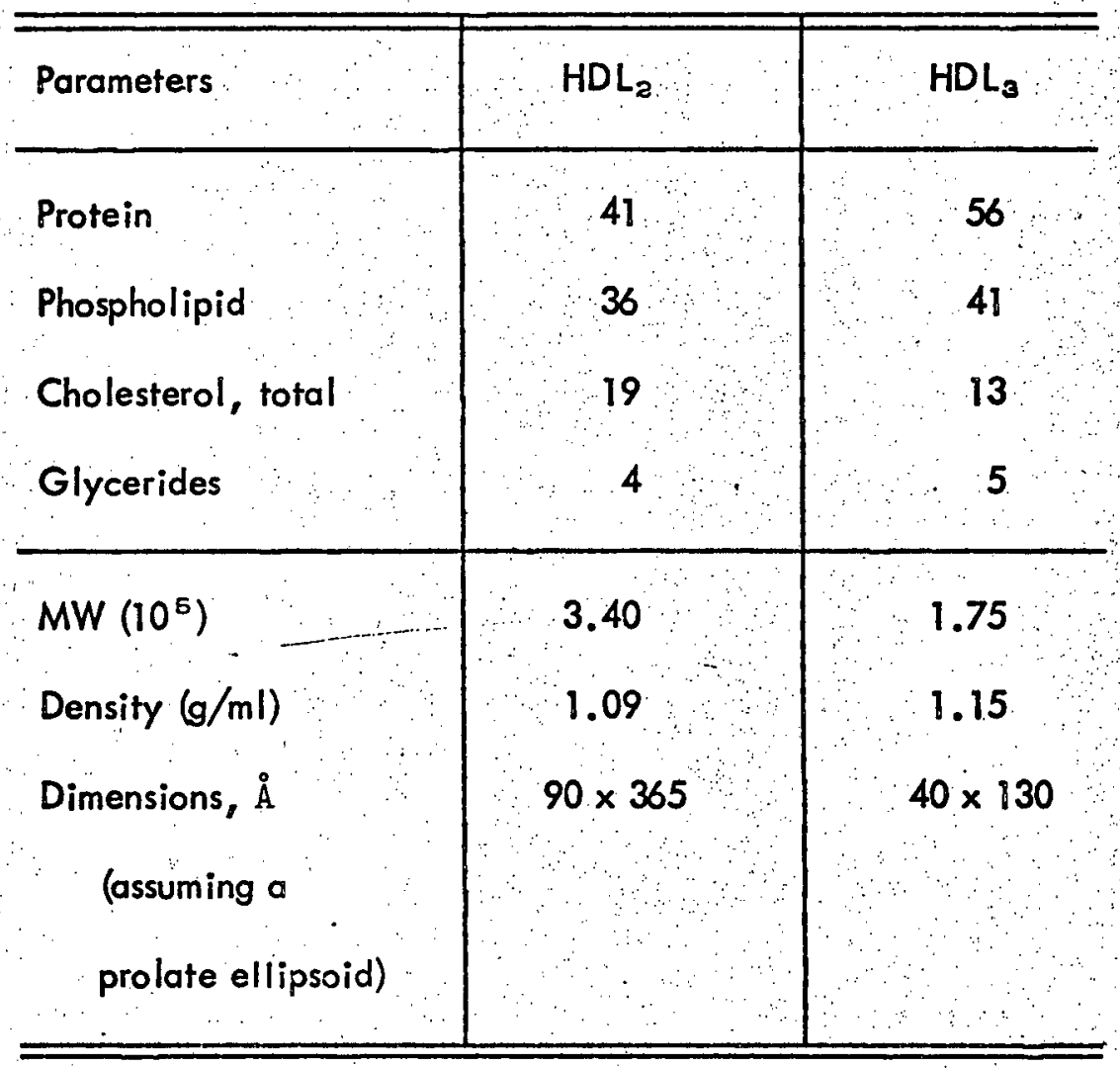


TABLE 2

CORRESPONDENCE OF MAJOR POLYPEPTIDE RRACTIONS

OF APO HDL BY THREE DIFFERENT PROCEDURES

\begin{tabular}{|c|c|c|c|}
\hline Authors & $\begin{array}{l}\text { Methods of } \\
\text { fractionation }\end{array}$ & Fractions & Characteristics \\
\hline Shore and Shore 16,17 & $\begin{array}{l}\text { DEAE-cellulose } \\
\text { chromatography }\end{array}$ & $4^{*}, 3 *$ & $\begin{array}{l}\text { 4-No isoleucine, } \\
\text { cysteine or cystine } \\
\text { 3-No histidine, argi- } \\
\text { nine and tryptophan }\end{array}$ \\
\hline Scanu et al. ${ }^{18}$ & $\begin{array}{l}\text { Sephadex G-200 } \\
\text { chromatography, } \\
8 \mathrm{M} \text { urea }\end{array}$ & III, IV & $\begin{array}{l}\text { III-Low isoleucine } \\
\text { cysteine or cystine } \\
\text { IV-Low histidine, argi- } \\
\text { nine and tryptophan }\end{array}$ \\
\hline Rudman ef al. ${ }^{19}$ & $\begin{array}{l}\text { Sephadex G-200 } \\
\text { chromatography, } \\
\text { I N acetic acid }\end{array}$ & II, III & $\begin{array}{l}\text { II and III same as III } \\
\text { and IV (see above) } \\
\quad \therefore\end{array}$ \\
\hline
\end{tabular}

*Fractions 3 and 4 are referred to by the authors as R-gln and R-thr based on their C-terminal analysis. Evidence that fractions III and IV are microheterogeneous has been recently obtained in this laboratory (Scanu, to be published). 